EDITORIAL

\title{
Small Exercise Breaks can Save your Brain from Prolonged Sitting
}

\author{
Eliza Prodel ${ }^{\circledR}$
}

Laboratory of Exercise Sciences, Fluminense Federal University, Niterói, RJ - Brazil

Editorial referring to the article: Effects of Breaking up Deskwork with Physical Activity Combined with Tea Consumption on Cerebrovascular Function, Mood, and Affect

A sedentary lifestyle is one of the major risk factors for the development of cardiovascular diseases. ${ }^{1}$ For instance, in 2019, stroke was the second-leading cause of death, as well as the primary cause of death and disability combined in the world. ${ }^{2}$ Active commuting to work is one strategy to decrease sedentary time and its impact, ${ }^{3}$ and the COVID-19 pandemic has changed work and daily routines worldwide. Moreover, the necessary social distancing has led to increased sedentary time, ${ }^{4}$ especially for those who are engaged in office desk work.$^{5}$

Acute prolonged sitting seems to lead to transient impairment of the endothelial function in the healthy population, ${ }^{6}$ whether this phenomenon is related to cardiovascular or cerebrovascular events is unknown. Nonetheless, prolonged sitting impairs the cerebrovascular function.,8 Conversely, exercise breaks from prolonged sitting seem to restore the endothelial ${ }^{9}$ and cerebrovascular function. ${ }^{7}$ Therefore, active breaks from prolonged sitting could be a good strategy to mitigate the effects of sedentary time. However, the ideal duration and intensity need further investigation. Additionally, the difficulty to implement exercise breaks as a daily routine for office workers must be considered.

In this sense, the effect of walk breaks from prolonged sitting on the cerebral vascular function was investigated, simulating a real-life routine. ${ }^{10}$ Hence desk workers took breaks from prolonged sitting to prepare tea every hour for six hours. ${ }^{1}$ After six hours of sitting, medium cerebral artery blood flow velocity $\left(\mathrm{MCB}_{\mathrm{v}}\right)$ decreased, and the small walk breaks (e.g.; five breaks of 150 meters of slow walking) were insufficient to re-establish $\mathrm{MCB}_{\mathrm{V}}$. The authors also observed an increased phase and reduced gain in very low frequency in cerebral autoregulation, which would indicate an improvement in cerebral autoregulation. However, the results concerning cerebral vasculature are difficult to interpret due to the decrease in the partial pressure of end-tidal carbon dioxide after the tea-break session $\left(\mathrm{PETCO}_{2}\right)$. The partial pressure of arterial carbon dioxide $\left(\mathrm{PaCO}_{2}\right)$ is the most potent regulator of cerebral blood flow, where small fluctuations evoke significant changes in cerebral blood flow. ${ }^{11}$

Furthermore, the walk breaks were followed by black tea consumption, but acute ingestion of tea seems to increase the endothelial function, ${ }^{12}$ and therefore the effect of the walk breaks cannot be isolated from the effect of the tea on the vascular function. Finally, the energy expenditure during those small walk breaks was not measured, but there is a possibility that higher energy expenditure is needed so that the exercise can exert a systemic vascular effect.

The study conducted by Speretta et al. ${ }^{10}$ does not allow a more thoughtful interpretation of the effect of small walk breaks or tea consumption on the possible cerebrovascular risk caused by prolonged sitting. However, the literature does support the conclusion that active breaks from a sedentary time are important in order to decrease vascular risks, but activities that produce higher energy expenditure than small walks must be considered.

\section{Keywords}

Exercise; Sedentarism; Lifestyle; Cerebrovascular Circulation; Stroke, Risk Factors; COVID-19; Pandemics; Physical Activity.

\section{Mailing Address: Eliza Prodel}




\section{References}

1. Lear SA, Hu W, Rangarajan S, Gasevic D, Leong D, Iqbal R, et al. The effect of physical activity on mortality and cardiovascular disease in 130000 people from 17 high-income, middle-income, and low-income countries: the PURE study. Lancet. 2017;390(10113):2643-54.

2. Global, regional, and national burden of stroke and its risk factors, 19902019: a systematic analysis for the Global Burden of Disease Study 2019. Lancet Neurol. 2021;20(10):795-820.

3. Dutheil F, Pelangeon S, Duclos M, Vorilhon P, Mermillod M, Baker JS, et al. Protective Effect on Mortality of Active Commuting to Work: A Systematic Review and Meta-analysis. Sports Med. 2020;50(12):2237-50.

4. Freiberg A, Schubert M, Romero Starke K, Hegewald J, Seidler A. A Rapid Review on the Influence of COVID-19 Lockdown and Quarantine Measures on Modifiable Cardiovascular Risk Factors in the General Population. Int J Environ Res Public Health. 2021;18(16):8567

5. Parry S, Straker L. The contribution of office work to sedentary behaviour associated risk. BMC Public Health. 2013;13:296.

6. Thosar SS, Bielko SL, Mather KJ, Johnston JD, Wallace JP. Effect of prolonged sitting and breaks in sitting time on endothelial function. Med Sci Sports Exerc. 2015;47(4):843-9.
7. Carter SE, Draijer R, Holder SM, Brown L, Thijssen DHJ, Hopkins ND Regular walking breaks prevent the decline in cerebral blood flow associated with prolonged sitting. J Appl Physiol (1985). 2018;125(3):790-8.

8. Burnet K, Blackwell J, Kelsch E, Hanson ED, Stone K, Fryer S, et al. Cerebrovascular function response to prolonged sitting combined with a high-glycemic index meal: A double-blind, randomized cross-over trial. Psychophysiology. 2021;58(8):e13830.

9. Paterson C, Fryer S, Zieff G, Stone K, Credeur DP, Barone Gibbs B, et al. The Effects of Acute Exposure to Prolonged Sitting, With and Without Interruption, on Vascular Function Among Adults: A Meta-analysis. Sports Med. 2020;50(11):1929-42.

10. Speretta GF, Fornasiero A, Johns JA, Hopkins N, Dick H. Thijssen DH, Low DA. Effects of Breaking up Deskwork with Physical Activity Combined with Tea Consumption on Cerebrovascular Function, Mood, and Affect. Int J Cardiovasc Sci. 2021; 34(6):644-653.

11. Hoiland RL, Fisher JA, Ainslie PN. Regulation of the Cerebral Circulation by Arterial Carbon Dioxide. Compr Physiol. 2019;9(3):1101-54.

12. Higashi Y. Coffee and Endothelial Function: A Coffee Paradox? Nutrients. 2019;11(9):2104. 